\title{
Retraction Note: Research and Application of Computer Control System Based on Complex Neural Network
}

Rui Yang

Published online: 14 November 2018

Retracted article: MATEC Web of Conferences 227, 02009 (2018)

https://doi.org/10.1051/matecconf/201822702009

We take a zero tolerance to any situation where fraudulent research is published in our journals. As a result, this article has been retracted by the Publisher because it is suspected to be a nonsensical publication computer-generated by the program SCIgen. 